\title{
NOAA/NGDC candidate models for the 12th generation International Geomagnetic Reference Field
}

\author{
Patrick Alken ${ }^{1,2^{*}}$, Stefan Maus ${ }^{1,2}$, Arnaud Chulliat ${ }^{1,2}$ and Chandrasekharan Manoj ${ }^{1,2}$
}

\begin{abstract}
The International Geomagnetic Reference Field (IGRF) is a model of the geomagnetic main field and its secular variation, produced every 5 years from candidate models proposed by a number of international research institutions. For this 12th generation IGRF, three candidate models were solicited: a main field model for the 2010.0 epoch, a main field model for the 2015.0 epoch, and the predicted secular variation for the five-year period 2015 to 2020. The National Geophysical Data Center (NGDC), part of the National Oceanic and Atmospheric Administration (NOAA), has produced three candidate models for consideration in IGRF-12. The 2010 main field candidate was produced from Challenging Minisatellite Payload (CHAMP) satellite data, while the 2015 main field and secular variation candidates were produced from Swarm and Ørsted satellite data. Careful data selection was performed to minimize the influence of magnetospheric and ionospheric fields. The secular variation predictions of our parent models, from which the candidate models were derived, have been validated against independent ground observatory data.
\end{abstract}

Keywords: Geomagnetic field; Magnetic field modeling; IGRF; Swarm

\section{Background}

The Earth's geomagnetic field is generated by a variety of sources. The primary source, known as the main field, is produced by the convection of liquid iron in the Earth's outer core. Additional sources include magnetized structures in the Earth's crust, as well as electric current systems flowing in the ionosphere and magnetosphere. The International Geomagnetic Reference Field (IGRF) is a standard representation of the Earth's geomagnetic main field and its secular variation, which is the temporal change of the magnetic field on time scales of 1 year or more. The IGRF is produced by an international collaborative effort and is updated every 5 years. For this 12 th generation IGRF, models were solicited from the international community for a retroactive update of the main field for 2010.0, a description of the main field for 2015.0, and the predicted secular variation of the main field for 2015.0 to 2020.0. Retroactive updates of the IGRF, such as the current call for 2010, are named as the Definitive

*Correspondence: patrick.alken@colorado.edu

${ }^{1}$ Cooperative Institute for Research in Environmental Science, University of Colorado, Boulder, CO, USA

${ }^{2}$ National Geophysical Data Center, Boulder, CO, USA
Geomagnetic Reference Field (DGRF), as it is possible to make use of more data around that epoch which was not available at the time of the original model release, and it is typically not planned to issue further updates of these past epochs.

While the IGRF is intended to describe the largescale, long wavelength part of the internal geomagnetic field, during the modeling process, it is necessary to carefully account for and parameterize the other sources so their effects can be separated from the final IGRF model. This usually involves careful data selection during geomagnetically quiet times to minimize the influence of external fields, parameterizing the steady ring-current field flowing in the magnetosphere and fitting the data to higher degree parent models, which would include shorter wavelength contributions from the Earth's magnetized crust. The final IGRF candidates are then extracted from these parent models by truncating the model expansions, most often parameterized by spherical harmonics, to the desired spatial resolution.

\section{Springer}

(c) 2015 Alken et al.; licensee Springer. This is an Open Access article distributed under the terms of the Creative Commons Attribution License (http://creativecommons.org/licenses/by/4.0), which permits unrestricted use, distribution, and reproduction in any medium, provided the original work is properly credited. 
In this paper, we describe the three National Geophysical Data Center (NGDC) candidate models for IGRF-12. Each candidate model was taken from a more extensive parent model, which attempted to parameterize the other various field sources described above. In section 'Candidate for the 2010 DGRF', we describe the parent model for our 2010 DGRF candidate. The methodology behind this model parameterization has been discussed in some detail in Maus et al. (2010) and Chulliat and Maus (2014), and so we only briefly review the main steps in producing the model. In section 'Main field and secular variation candidates for IGRF 2015', we discuss our candidate models for the 2015 IGRF main field and secular variation, which were both extracted from the same parent model. For these models, we used a similar methodology to the 2010 DGRF; however, there are some differences in the data selection and model parameterization, and the underlying data sets rely on different satellites, and so we provide a more complete discussion of these two models.

\section{Methods}

\section{Candidate for the 2010 DGRF}

The NGDC candidate for the 2010 Definitive Geomagnetic Reference Field was based entirely on observations from the Challenging Minisatellite Payload (CHAMP) satellite (Reigber et al. 2003). CHAMP was launched in July 2000 into a circular, near-polar orbit with an inclination of $87^{\circ}$. CHAMP's initial altitude was $454 \mathrm{~km}$, which decayed to about $250 \mathrm{~km}$ by the end of its mission in September 2010. A series of geomagnetic main field models were derived from CHAMP in order to study the core secular acceleration from 2000 to 2010. More complete details of these models can be found in Chulliat and Maus (2014) and Maus et al. (2010), but we will briefly summarize their construction here. First, the CHAMP data were separated into half-orbital tracks, and an root-meansquare (rms) difference was computed for each track with respect to the POMME-6 main field model (Maus et al. 2010). Tracks with an rms difference greater than $3 \mathrm{nT}$ were discarded. The CHAMP data were downsampled to one measurement every $20 \mathrm{~s}$ and additionally corrected for ocean tidal fields (Kuvshinov and Olsen 2005), diamagnetic currents originating in the ionosphere (Lühr et al. 2003), and external magnetospheric fields (Lühr et al. 2010). The data were then divided into three categories: (a) spanning $-90^{\circ}$ to $-50^{\circ}$ geomagnetic latitude, (b) spanning $-60^{\circ}$ to $+60^{\circ}$ geomagnetic latitude, and (c) spanning $+50^{\circ}$ to $+90^{\circ}$ geomagnetic latitude. The following data selection criteria were applied independently to each of the three datasets to select for geomagnetically quiet times. In the following list, mid latitudes refer to the region between $-60^{\circ}$ to $+60^{\circ}$ geomagnetic latitude, while high latitudes refer to the regions above $50^{\circ}$ and below $-50^{\circ}$ geomagnetic latitude.

1. Dst index magnitude does not exceed $30 \mathrm{nT}$ at all latitudes.

2. $\left|\frac{d}{d t} D s t\right| \leq 2 \mathrm{nT} / \mathrm{h}$ at mid latitudes, $5 \mathrm{nT} / \mathrm{h}$ at high latitudes.

3. $a_{m}$ index less than 12 at mid latitudes, 27 at high latitudes.

4. $a_{m} 3 \mathrm{~h}$ previous less than 15 at mid latitudes, 27 at high latitudes.

5. Interplanetary magnetic field components: $\left|B_{y}\right| \leq 8$ $\mathrm{nT},-2 \leq\left|B_{z}\right| \leq 6 \mathrm{nT}$.

6. $\left|E_{m}\right| \leq 0.8 \mathrm{mV} / \mathrm{m}$.

7. In the mid latitude region, vector and scalar data between 0500 and 2000 local time were discarded. At high latitudes, no vector data is used, but scalar data at all local times is included.

For each of the models used in the study of Chulliat and Maus (2014), the Gauss coefficients of the magnetic scalar potential were parameterized as a second order Taylor series in time, with the first derivative corresponding to the linear secular variation and the second derivative corresponding to the secular acceleration. Models were computed using a sliding window which included 3 years of CHAMP data. While a 3-year window introduces some time averaging of the resulting coefficients, it was found that this length was necessary to obtain stable estimates of the secular variation and secular acceleration parameters. Damping was applied to the secular variation coefficients above degree 13 and secular acceleration coefficients above degree 8 . All coefficients were estimated using least squares minimization of the data residuals. The latest model estimated from this method had an epoch of 2009.17. For our final DGRF candidate, we extrapolated the main field coefficients to epoch 2010.0 using the secular variation and secular acceleration coefficients and the second order Taylor series relation. Since this modeling method has been reported previously, we refer the reader to Maus et al. (2010) for further discussion and plots of the model residuals and their statistics.

Main field and secular variation candidates for IGRF 2015 For the 2015.0 epoch, NGDC has produced candidate models for both the main field and secular variation.

\section{Data sources}

We exclusively used satellite observations from the Ørsted and Swarm missions to construct our IGRF candidate models for the 2015.0 epoch. Ørsted (Olsen et al. 2003) was launched in February 1999 into an elliptical near-polar orbit with an inclination of $96.4^{\circ}$. Its 
current perigee and apogee are about 635 and $835 \mathrm{~km}$, respectively, and its orbital period is about $99.5 \mathrm{~min}$. Ørsted has been providing only scalar field measurements since 2005 due to a star camera failure. It has furthermore ceased providing data after June 2013. While only half a year of scalar data from Ørsted was available in 2013, we found that including this data in the modeling helped to resolve the higher degree secular variation coefficients.

The Swarm satellite mission (Friis-Christensen et al. 2006) is composed of three satellites which were launched in November 2013 into near-polar orbits. Each Swarm satellite carries a vector fluxgate magnetometer (VFM) instrument in addition to an absolute scalar magnetometer (ASM) (Leger et al. 2009) which provides the scalar reference for calibrating the vector measurement. A lower pair of satellites (Alpha and Charlie) fly in a side-by-side constellation with an inclination of $87.4^{\circ}$ and an altitude of about $465 \mathrm{~km}$. The third satellite (Bravo) flies in a higher orbit of about $520 \mathrm{~km}$ with an inclination of $88^{\circ}$.

\section{Data selection}

For the main field and secular variation models for epoch 2015.0, we include all available Ørsted and Swarm data from January 2013 through September 2014, downsampled to 1 measurement every $20 \mathrm{~s}$. We performed an initial quality control on the datasets by separating the data into half-orbit tracks and computing along-track rms differences with the IGRF-11 (Finlay et al. 2010) main field model. Tracks with large rms differences were discarded. Causes for large differences could include orbit maneuvers performed in the first few months of the Swarm mission, uncorrected stray spacecraft fields, or anomalous measurements. We also filtered Swarm data according to the following flags criteria:

- Flags_F < 64 (ASM instrument recorded data)

- Flags_B $<255$ (VFM instrument recorded data)

- Flags_q $<31$ (at least 2 star cameras operational)

No flag selection on Ørsted data was performed. We additionally used the following data selection criteria to attempt to minimize the presence of magnetospheric and ionospheric fields:

1. Dst index magnitude does not exceed $30 \mathrm{nT}$.

2. Interplanetary magnetic field components: $\left|B_{y}\right| \leq 8$ $\mathrm{nT},-2 \leq B_{z} \leq 6 \mathrm{nT}$.

3. Ap index less than 12 below $60^{\circ}$ geomagnetic latitude.

4. Ap index less than 27 above $60^{\circ}$ geomagnetic latitude.

5. Local times between 0500 and 2200 are excluded below $60^{\circ}$ geomagnetic latitude for Swarm data. For Ørsted, we exclude local times between 0500 and
2000 , since the satellite is near a $9 \mathrm{am} / 9 \mathrm{pm}$ orbit for the spring of 2013.

6. Above $60^{\circ}$ geomagnetic latitude, the sun must be at least $10^{\circ}$ below the horizon to ensure darkness.

7. Vector data (from Swarm) is used only below $55^{\circ}$ geomagnetic latitude to reduce contamination from high-latitude ionospheric current systems. Scalar data (from Swarm and Ørsted) is used at all latitudes.

Using this data selection criteria, we show in Figure 1 the temporal distribution of scalar (top) and vector (bottom) data used for the modeling. There is a large gap after 2013.5 due to a lack of Ørsted measurements. There are also two smaller gaps during the Swarm time frame, due to the satellites entering local times which are excluded according to our criteria above.

\section{Model parameterization}

Once the final dataset was prepared according to the selection criteria discussed above, we fit a parent geomagnetic main field model to the dataset. In order to minimize influences from crustal and external fields, we remove a priori field models of these sources from the data. For the crustal field, we subtract the MF7 lithospheric field model. The MF7 model used a very similar modeling methodology to the MF6 model (Maus et al. 2008); however, it used more recent CHAMP measurements, when CHAMP was flying at a lower altitude during solar minimum and also extends the crustal field resolution from spherical harmonic degree 120 to 133 . For external sources, we subtract the POMME- 6 external field model (Lühr et al. 2010; Maus and Lühr 2005). To fit the remain-

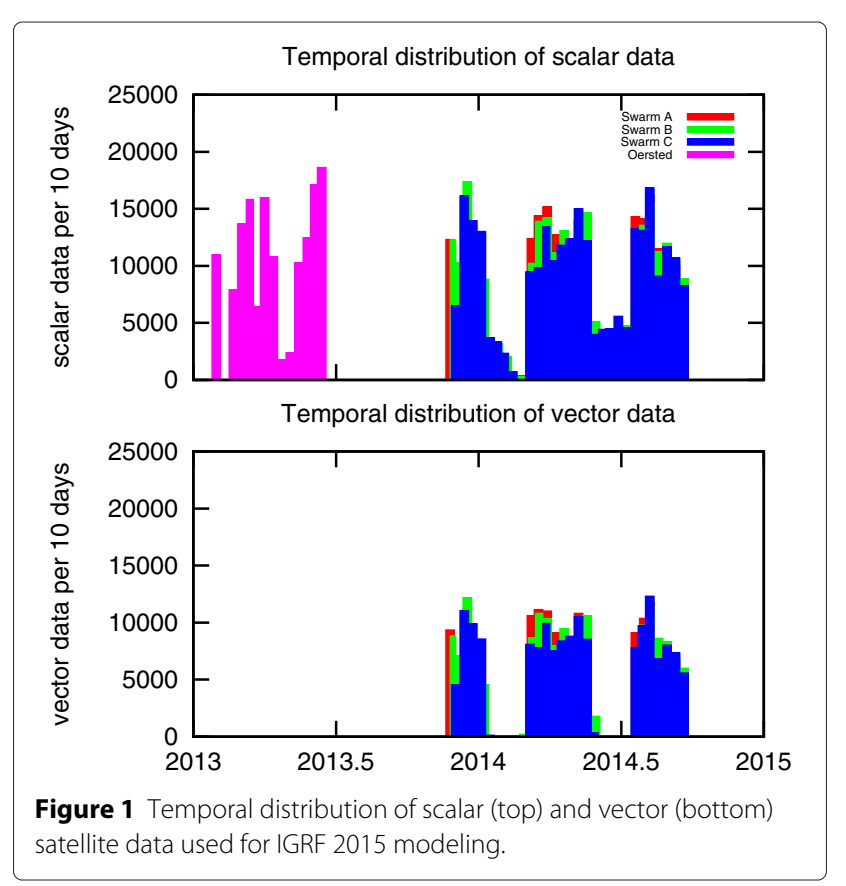


ing internal main field, we expand the magnetic scalar potential as

$$
\begin{aligned}
V^{\mathrm{int}}(r, \theta, \phi)= & a \sum_{n=1}^{N}\left(\frac{a}{r}\right)^{n+1} \sum_{m=0}^{n} P_{\mathrm{nm}}(\cos \theta) \\
& \times\left(g_{\mathrm{nm}}(t) \cos m \phi+h_{\mathrm{nm}}(t) \sin m \phi\right)
\end{aligned}
$$

where $a=6371.2 \mathrm{~km}$ is a reference radius, $N$ is the maximum spherical harmonic degree, $P_{\mathrm{nm}}(\cos \theta)$ is the Schmidt-normalized associated Legendre function, and $g_{\mathrm{nm}}(t), h_{\mathrm{nm}}(t)$ are the time-dependent Gauss coefficients to be determined. The Gauss coefficients are expanded to first-order in time as

$$
\begin{aligned}
& g_{\mathrm{nm}}(t)=g_{\mathrm{nm}}\left(t_{0}\right)+\dot{g}_{\mathrm{nm}}\left(t_{0}\right)\left(t-t_{0}\right) \\
& h_{\mathrm{nm}}(t)=h_{\mathrm{nm}}\left(t_{0}\right)+\dot{h}_{\mathrm{nm}}\left(t_{0}\right)\left(t-t_{0}\right)
\end{aligned}
$$

The model epoch was chosen as $t_{0}=2014.3$ corresponding to the mean of the timestamps in the final dataset. For the parent model, we fit the main field to spherical harmonic degree 20 and the secular variation to degree 12. In addition to estimating the internal Gauss coefficients, we coestimate Swarm Euler angles, representing a $3 \mathrm{D}$ rotation from the fluxgate magnetometer frame into the star camera frame. We compute these angles in bins of 30 days for each of the three Swarm satellites. Finally, due to uncertainties in the Dst index baseline, which drives the POMME external field model, we fit an additional daily correction to the external field model represented as an external dipole oriented in the internal main field dipole direction, as well as a smaller-induced counterpart. To estimate all these parameters, we define a penalty function of residuals as

$$
\chi^{2}(\mathbf{g} ; \mathbf{k} ; \alpha, \beta, \gamma)=\sum_{i=1}^{N_{\text {vec }}} \boldsymbol{\epsilon}_{i} \cdot \boldsymbol{\epsilon}_{i}+\sum_{i=1}^{N_{\text {scal }}} f_{i}^{2}
$$

where $N_{\text {vec }}=505,924$ is the total number of vector measurements, $N_{\text {scal }}=934,878$ is the total number of scalar measurements, $\mathbf{g}$ is a vector of main field and secular variation Gauss coefficients, $\mathbf{k}$ is a vector of daily corrections to the external field model, and $\alpha, \beta, \gamma$ are the Swarm Euler angles. The vector residuals are defined as

$$
\boldsymbol{\epsilon}_{i}=R_{q} R_{3}(\alpha, \beta, \gamma) \mathbf{B}_{i}^{V F M}-\mathbf{B}^{\text {model }}(\mathbf{g}, \mathbf{k}),
$$

where $\mathbf{B}_{i}^{V F M}$ is the Swarm vector measurement in the instrument (VFM) frame, $R_{3}(\alpha, \beta, \gamma)$ represents the 3D rotation matrix to rotate from the VFM to the star camera frame using Euler angles $\alpha, \beta, \gamma$, and $R_{q}$ is the rotation matrix from the star camera frame to NorthEast-Center (NEC) geographic coordinates (see Olsen et al. 2013, equations. 1 to 3 ). $\mathbf{B}^{\text {model }}$ is our magnetic field model in the NEC frame, which is specified by the Gauss coefficients $\mathbf{g}$ and daily external field corrections $\mathbf{k}$ (see below). The scalar residuals are defined as

$$
f_{i}=F_{i}-\left|\mathbf{B}^{\text {model }}(\mathbf{g}, \mathbf{k})\right|,
$$

where $F_{i}$ are the scalar field measurements from both Swarm and Ørsted. The field model is defined as

$$
\begin{aligned}
\mathbf{B}^{\text {model }}(\mathbf{g}, \mathbf{k})= & \mathbf{B}^{\text {int }}(\mathbf{g})+\mathbf{B}^{\text {crust }}+\mathbf{B}^{\text {ext,pomme }} \\
& +\mathbf{B}^{\text {ext,correction }}(\mathbf{k})
\end{aligned}
$$

where $\mathbf{B}^{\text {int }}=-\nabla V^{\text {int }}, \mathbf{B}^{\text {crust }}$ is the MF7 crustal field for spherical harmonic degrees 16 to 133 , $\mathbf{B}^{\text {ext,pomme }}$ is the POMME-6 external field, and $\mathbf{B}^{\text {ext,correction }}$ is the daily external correction, defined as

$$
\mathbf{B}^{\text {ext,correction }}(\mathbf{k})=k(t)\left(0.7 \hat{\mathbf{B}}^{\text {ext,dipole }}+0.3 \hat{\mathbf{B}}^{\text {int,dipole }}\right)
$$

Here, $k(t)$ is the magnitude of the correction in nT, with one value for each day of available data. The dimensionless $n=1$ external and internal dipole fields are given by

$$
\begin{aligned}
& \hat{\mathbf{B}}^{\text {ext,dipole }}=-a \nabla \hat{V}^{\text {ext,dipole }} \\
& \hat{\mathbf{B}}^{\text {int,dipole }}=-a \nabla \hat{V}^{\text {int,dipole }}
\end{aligned}
$$

with dimensionless scalar potentials

$$
\begin{aligned}
\hat{V}^{\text {ext,dipole }}(r, \theta, \phi)= & \sum_{m=0}^{1}\left(\frac{r}{a}\right) P_{1 m}(\cos \theta)\left(\hat{g}_{1 m} \cos m \phi\right. \\
& \left.+\hat{h}_{1 m} \sin m \phi\right)
\end{aligned}
$$

and

$$
\begin{aligned}
\hat{V}^{\text {int,dipole }}(r, \theta, \phi)= & \sum_{m=0}^{1}\left(\frac{a}{r}\right)^{2} P_{1 m}(\cos \theta) \\
& \times\left(\hat{g}_{1 m} \cos m \phi+\hat{h}_{1 m} \sin m \phi\right)
\end{aligned}
$$

Finally, the dimensionless dipole coefficients are given by

$$
\begin{aligned}
& \hat{g}_{1 m}=\frac{g_{1 m}}{\sqrt{g_{10}^{2}+g_{11}^{2}+h_{11}^{2}}} \\
& \hat{h}_{11}=\frac{h_{11}}{\sqrt{g_{10}^{2}+g_{11}^{2}+h_{11}^{2}}}
\end{aligned}
$$


i.e., along the internal dipole direction. The values 0.7 and 0.3 are approximate relative strengths of the external and induced fields (Maus and Weidelt 2004).

We expand the parent model main field to degree 20 and secular variation to degree 12 , for a total of $440+168=$ 608 internal field coefficients. The Euler angles are calculated in bins of 30 days, and so there are 11 sets of angles computed for each satellite during the data period, leading to $(3$ satellites $) \times(3$ angles $) \times 11=99$ Euler angles. Finally, there are a total of 375 days of data used in the model and so 375 external field correction coefficients. The total number of parameters estimated is therefore $608+99+375=1082$. Since the vector residuals are nonlinear in the Euler angles $\alpha, \beta, \gamma$, and the scalar residuals are nonlinear in both $\mathbf{g}$ and $\mathbf{k}$, the model parameters are estimated using iteratively reweighted nonlinear least squares minimization of the penalty function $\chi^{2}$, using Huber weights at each iteration (Huber 1996) to reduce the effect of data outliers. We found that a total of five iterations were sufficient for convergence of the method. Since the polar regions are sampled more often than middle and low latitudes, we bin the data into $1.8^{\circ}$ latitude by $3.6^{\circ}$ longitude bins and assign initial weights to each data point according to

$$
w_{\mathrm{ij}}=\frac{1}{K} \sqrt{\frac{a_{\mathrm{ij}}}{n_{\mathrm{ij}}}}
$$

where $a_{\mathrm{ij}}, n_{\mathrm{ij}}$ are the area on a unit sphere and number of measurements for bin $(i, j)$, respectively. This expression effectively up-weights sparsely sampled regions with larger areas (typically low latitudes) and down-weights densely sampled regions with smaller areas (typically at the poles). $K$ is a normalization constant chosen so that $\sum_{\mathrm{ij}} w_{\mathrm{ij}}=1$. At each step of the iteration, these initial weights are multiplied by the Huber weights to produce the final weights.

\section{Results and discussion}

The residual statistics are shown in Table 1. We find the Swarm vector residuals have low means with rms errors of around $4 \mathrm{nT}$ or less. The Swarm scalar residuals below $55^{\circ}$ geomagnetic latitude have near-zero means with rms differences of about $2.6 \mathrm{nT}$, while the Swarm scalar residuals above $55^{\circ}$ have higher means and rms errors, due primarily to unmodeled currents in the polar region. The Ørsted scalar residuals show higher means and rms differences than Swarm for both latitude regions. This is likely due to the significantly fewer Ørsted data used in the model, compared to Swarm, and the difficulty in modeling the change of the main field into 2013 using only a linear secular variation parameterization. We plot in Figure 2 spatial maps of the residuals from the scalar and vector measurements from the three Swarm satellites against the parent model. We don't find any significant spatial structure in the residuals, indicating a good fit to the Swarm data. Figure 3 shows the scalar residuals from Ørsted. Here we see a negative residual band at the equator, with positive bands at higher latitudes in both the northern and southern hemisphere. This indicates that the model is overestimating the Ørsted data in the equatorial region, while underestimating at higher latitudes. This could be due to some unmodeled external field which is more prominent at Ørsted altitude than for Swarm. Another possibility is systematic differences between the Swarm and Ørsted datasets. While it is difficult to determine the root cause of this discrepancy, Maus et al. (2010) found a similar effect in comparing the residuals of Ørsted and CHAMP to a parent main field model.

\section{Candidate models}

After the parent model was estimated from the satellite data, we truncated it to spherical harmonic degree 13 for the main field and degree 8 for the secular variation, as specified in the IGRF call. We found that using 10 months of Swarm data alone was only able to provide an undamped estimate of the secular variation (SV) to degree 6. Including the 6 months of Ørsted data in 2013 allows an undamped SV estimate to degree 11. Since the parent model was computed for the epoch 2014.3, we advanced the main field coefficients to 2015.0 using the linear secular variation. The secular variation coefficients remained unchanged since we did not include higher order time derivatives in the model.

Table 1 Residual statistics of satellite measurements against parent model

\begin{tabular}{|c|c|c|c|c|c|c|c|c|}
\hline & \multicolumn{2}{|c|}{ Swarm A } & \multicolumn{2}{|c|}{ Swarm B } & \multicolumn{2}{|c|}{ Swarm C } & \multicolumn{2}{|c|}{$\varnothing$ rsted } \\
\hline & Mean & Rms & Mean & Rms & Mean & Rms & Mean & Rms \\
\hline$B_{x}$ & -0.04 & 3.81 & 0.14 & 4.00 & 0.29 & 4.01 & & \\
\hline By & -0.11 & 3.33 & -0.08 & 3.32 & -0.15 & 3.35 & & \\
\hline$B_{z}$ & 0.07 & 1.81 & 0.04 & 2.07 & -0.12 & 2.06 & & \\
\hline Low-latitude $|\mathbf{B}|$ & 0.01 & 2.59 & 0.08 & 2.60 & 0.09 & 2.64 & 0.56 & 5.16 \\
\hline High-latitude |B| & 0.84 & 11.79 & 1.26 & 11.14 & 1.20 & 11.88 & 1.94 & 15.59 \\
\hline
\end{tabular}

Low-latitude is defined as below $55^{\circ}$ geomagnetic latitude where vector data are used, while high-latitude is defined as above $55^{\circ}$. All values are in units of $\mathrm{nT}$. 


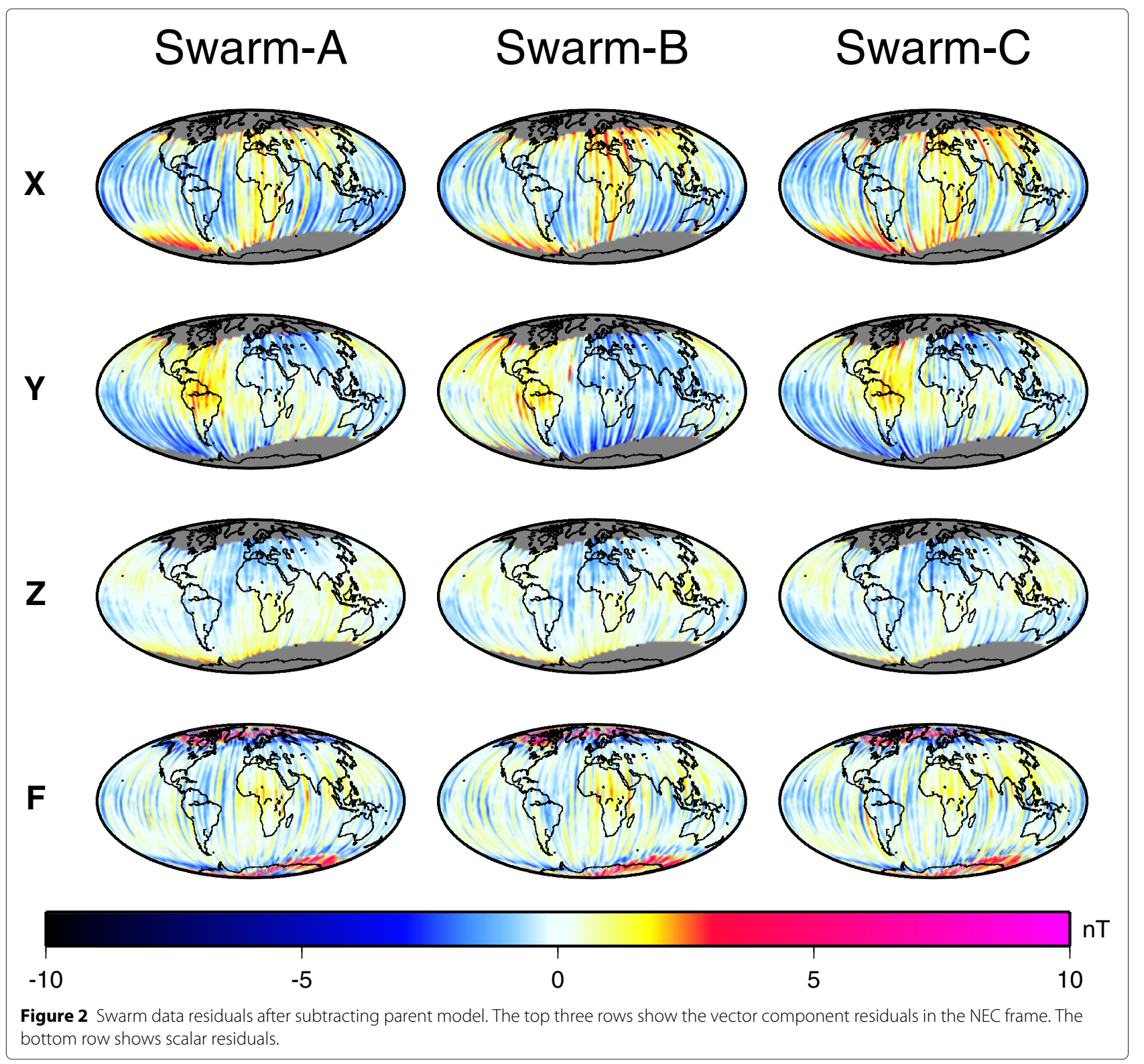

\section{Validation with ground observatories}

As an independent validation, we compare our IGRF-12 candidate secular variation model with a global set of geomagnetic observatory data. We use an hourly mean database of geomagnetic observatory data compiled by the British Geological Survey for years 2010 to 2014. A total of 66 geomagnetic observatories, distributed across the world, were available for the analysis with data at least up to June 2014. To estimate the secular variation at each observatory, we fit cubic splines with knots separated by 1 year separately to the geomagnetic X, Y, and $\mathrm{Z}$ components in a least-square sense. Secular variation was then determined by subtracting the spline value at year 2013.8 from that at 2014.8, giving an SV estimate at the date 2014.3, which was the same epoch used in the field modeling. We then find the global mean and rms of the differences between the model and prediction. The results are shown in Table 2. The rms errors were calculated after the global means were removed from all the differences. For a reference, we also provide comparison with SV values predicted by the previous IGRF model, IGRF-11 at 2014.3. As expected, the rms errors with NGDC's IGRF-12 candidate model are lower than those with the IGRF-11 prediction for all the components. The larger values for the mean of differences between the predicted and observed X component is most likely due 


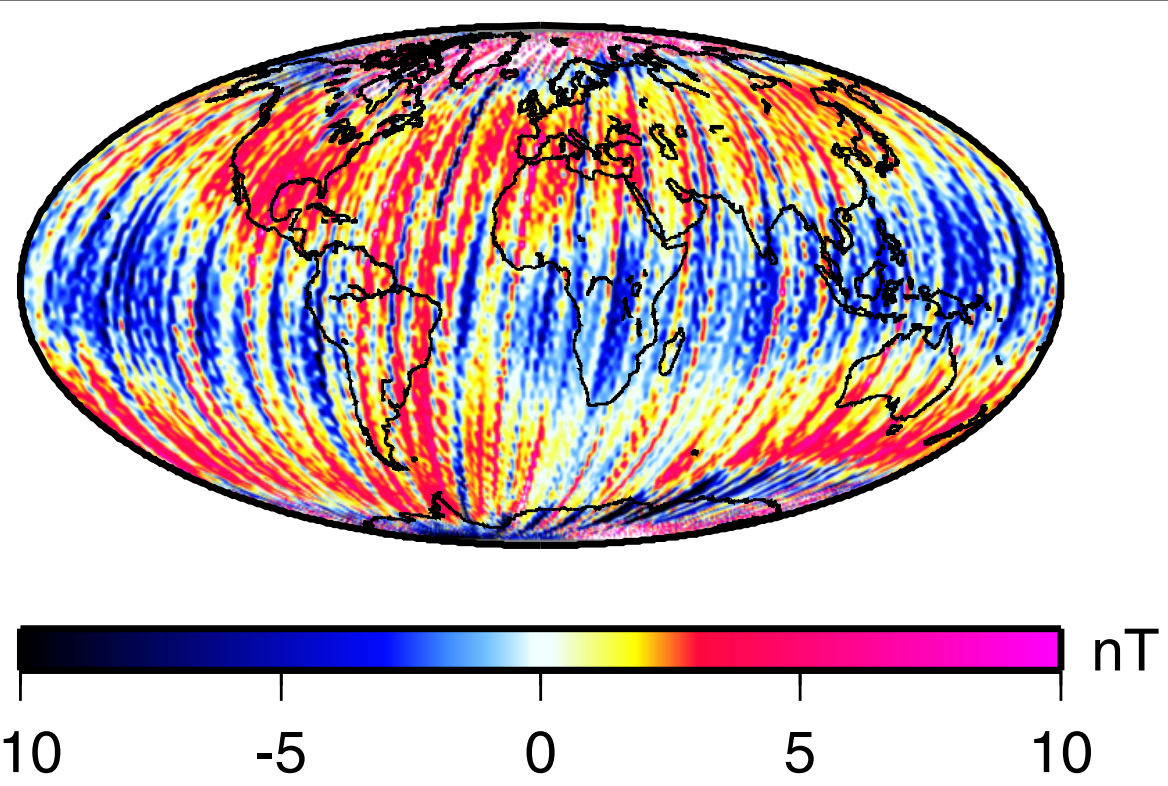

Figure $3 \varnothing$ rsted scalar data residuals after subtracting parent model.

to the uncorrected steady change of the external field in the observatory data, which manifests most strongly in the north/south direction.

In a second validation approach, we compared annual differences of monthly means at selected observatories with predictions of our candidate SV model for 2015.0. We also compared them with the SV obtained by taking the differences between our candidate main field models for 2010.0 and 2015.0, i.e., the average SV in 2012.5 based on these two models and with the SV model obtained as a by-product of the 2010 candidate calculation. Observatory monthly means were calculated from nighttime (between 2200 and 0500 LT) and geomagnetically quiet $\left(K_{p}<2\right.$ and $\left.\left|D_{s t}\right|<20 \mathrm{nT}\right)$ hourly mean values at six observatories of the Bureau Central de Magnétisme Terrestre (www.bcmt.fr) network: Mbour (MBO, Senegal), Tamanrasset (TAM, Algeria), Chambon-la-forêt (CLF, France), Kourou (KOU, French Guiana), Phu Thui (PHU, Vietnam), and Pamatai (PPT, Tahiti). Quasi-definitive data (Peltier and Chulliat 2010) were used from January to July 2014; definitive data were used before 2014. Figure 4 shows the results at two observatories, $\mathrm{MBO}$ and $\mathrm{KOU}$,

Table 2 Comparison of IGRF-11 and our IGRF-12 candidate SV models with spline fits to 66 observatories

\begin{tabular}{|c|c|c|c|c|c|c|}
\hline & \multicolumn{2}{|c|}{$\mathrm{dX} / \mathrm{dt}$ (nT/year) } & \multicolumn{2}{|c|}{$\mathrm{dY} / \mathrm{dt}$ (nT/year) } & \multicolumn{2}{|c|}{$\mathrm{dZ} / \mathrm{dt}$ (nT/year) } \\
\hline & Mean & Rms & Mean & Rms & Mean & Rms \\
\hline IGRF-11 & -8.96 & 14.20 & 1.25 & 9.93 & 3.85 & 18.15 \\
\hline $\begin{array}{l}\text { IGRF-12 } \\
\text { candidate }\end{array}$ & -11.82 & 10.31 & 1.19 & 4.77 & 2.12 & 7.91 \\
\hline
\end{tabular}

located in the area where large, anti-correlated secular acceleration pulses have been occurring since 2006 (Chulliat and Maus 2014). Geomagnetic jerks marked the beginning and end of each pulse, in 2003, 2007 and 2011, as can be seen in the azimuthal and radial components at both observatories. Jerks (and associated pulses) are the biggest obstacles to a successful forecasting of the secular variation over a few years.

We found that the candidate SV model for 2015.0 was in agreement with the latest trends of the $\mathrm{SV}$ at each observatory. For example, at KOU, the latest data available show a strong acceleration of the azimuthal component after 2012 which is captured by our 2015.0 SV model. At $\mathrm{MBO}$, the model is further away from the observatory data on both the azimuthal and radial component, but is in agreement with a reasonable extrapolation of the SV trends before 2014. We also check that the $2010 \mathrm{SV}$ model is in very good agreement with the selected observatory data, despite the extrapolation from 2009.17 to 2010.0. As this validation is made retrospectively, i.e., after acquiring enough observatory data to recover the SV around 2010.0, this gives us confidence in the extrapolation applied to the 2015.0 SV candidate. This figure also illustrates the effect of the truncation of an SV model from degree 13 to degree 8; the prediction from the truncated degree 8 SV at 2010.0 are a few (less than 5) nT/year away from the SV measured at the selected observatories. Finally, the average SV in 2012.5 is found to be in agreement with the average SV from observatory data over 2010 to 2015 thus further validating our main field candidate models. Similar results were found at the other four observatories. None of our candidate models included observatory data 

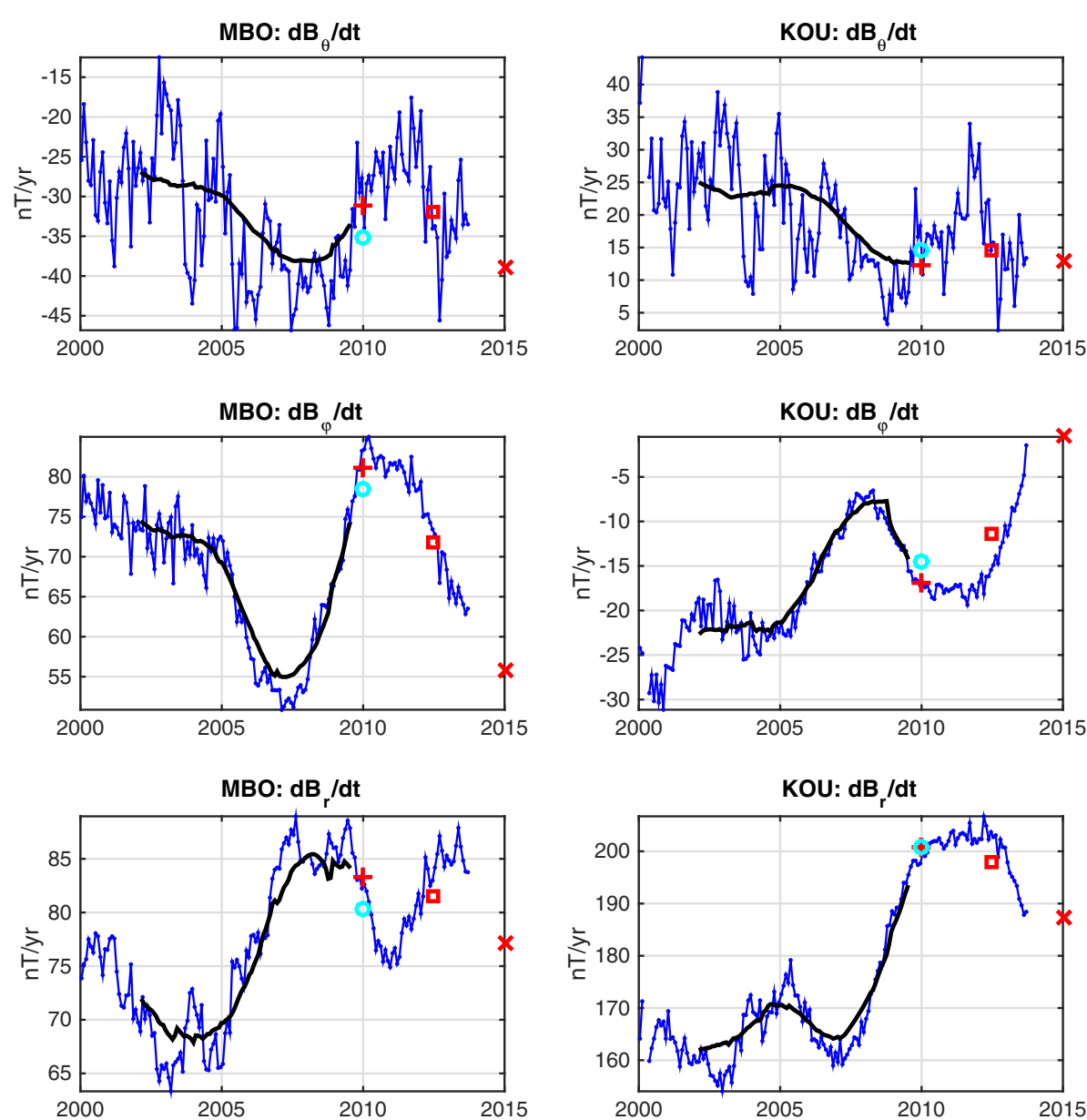

Figure 4 Validation of candidate DGRF and IGRF models against independent observatory data. Annual differences between the field at $\mathrm{t}+6$ months and t -6 months (blue dots and lines) between quiet monthly means at the MBO and KOU observatories are compared to predictions of the candidate SV models. The DGRF parent SV model for 2010.0 is shown truncated at degree 13 (red crosses) and degree 8 (cyan circles). The IGRF parent SV model for 2015.0 is shown truncated at degree 8 (red X). The average SV over 2010.0 to 2015.0, computed from differences of the DGRF and IGRF main field candidates, are shown as red squares. The predictions by the series of SV models calculated in Chulliat and Maus (2014) are also represented as black lines.

in their calculation; therefore, this analysis represents a test against truly independent data.

\section{Conclusions}

The National Geophysical Data Center at National Oceanic and Atmospheric Administration (NOAA) has produced three candidate models for the 12th generation IGRF. We provide a 2010 DGRF main field candidate based on CHAMP satellite data, a 2015 IGRF main field candidate and a 2015 to 2020 secular variation candidate based on Swarm and Ørsted satellite data. These candidates were all derived from sophisticated parent models which include careful data selection, high degree crustal field parameterization, and external magnetospheric field corrections. The resulting models were truncated to spherical harmonic degree 13 for the main field and degree 8 for the secular variation. All three candidate models have been successfully validated against independent ground observatory data.

\section{Competing interests}

The authors declare that they have no competing interests.

\section{Authors' contributions}

SM produced the 2010 DGRF main field candidate. PA produced the 2015 IGRF main field and 2015-2020 IGRF secular variation candidates and drafted the manuscript. AC and CM performed the validations against independent observatory data. All authors read and approved the final manuscript.

\section{Acknowledgements}

The support of the CHAMP mission by the German Aerospace Center (DLR) and the Federal Ministry of Education and Research is gratefully

acknowledged. The Ørsted mission was extensively supported by the Danish Government, NASA, ESA, CNES, and DARA. The authors acknowledge ESA for providing access to the Swarm $\mathrm{L} 1 \mathrm{~b}$ data. The results presented in this paper 
rely on data collected at magnetic observatories. We thank the national institutes that support them and INTERMAGNET for promoting high standards of magnetic observatory practice (www.intermagnet.org).

\section{Received: 30 January 2015 Accepted: 19 March 2015}

Published online: 12 May 2015

\section{References}

Chulliat A, Maus S (2014) Geomagnetic secular acceleration, jerks, and a localized standing wave at the core surface from 2000 to 2010. J Geophys Res Solid Earth 119:1531-1543. doi:10.1002/2013JB010604

Finlay CC, Maus S, Beggan CD, Bondar TN, Chambodut A, Chernova TA, Chulliat A, Golovkov VP, Hamilton B, Hamoudi M, Holme R, Hulot G, Kuang W, Langlais B, Lesur V, Lowes FJ, Lühr H, Macmillan S, Mandea M, McLean S, Manoj C, Menvielle M, Michaelis I, Olsen N, Rauberg J, Rother M, Sabaka TJ, Tangborn A, Tøffner-Clausen L, Thébault E, et al (2010) International geomagnetic reference field: the eleventh generation. Geophys J Int 183:1216-1230. doi:10.1111/j.1365-246X.2010.04804.x. Issue 3

Friis-Christensen E, Lühr H, Hulot G (2006) Swarm: a constellation to study the Earth's magnetic field. Earth Planets Space 58:351-358

Huber PJ (1996) CBMS-NSF regional conference series in applied mathematics. Society for Industrial and Applied Mathematics, Philadelphia

Kuvshinov A, Olsen N (2005) 3-D modelling of the magnetic fields due to ocean tidal flow. In: Reigber C, Lühr H, Schwintzer P, Wickert J (eds). Earth Observation with CHAMP: results from three years in Orbit. Springer, Berlin. pp 359-365

Leger J-M, Bertrand F, Jager T, Prado ML, Fratter I, Lalaurie J-C (2009) Swarm absolute scalar and vector magnetometer based on helium 4 optical pumping. Procedia Chem 1(1):634-637. doi:10.1016/j.proche.2009.07.158. Proceedings of the Eurosensors XXIII conference

Lühr H, Rother M, Maus S, Mai W, Cooke D (2003) The diamagnetic effect of the equatorial Appleton anomaly: its characteristics and impact on geomagnetic field modeling. Geophys Res Lett 30(17). doi:10.1029/2003GL017407

Lühr H, Maus S (2010) Solar cycle dependence of quiet-time magnetospheric currents and a model of their near-Earth magnetic fields. Earth Planets Space 62:843-848

Maus S, Weidelt P (2004) Separating the magnetospheric disturbance magnetic field into external and transient internal contributions using a 1D conductivity model of the Earth. Geophys. Res. Lett. 31(12). doi:10.1029/2004GL020232

Maus S, Lühr H (2005) Signature of the quiet-time magnetospheric magnetic field and its electromagnetic induction in the rotating Earth. Geophys J Int 162:755-763. doi:10.1111/j.1365-246X.2005.02691.x

Maus S, Manoj C, Rauberg J, Michaelis I, Lühr H (2010) NOAA/NGDC candidate models for the 11th generation International Geomagnetic Reference Field and the concurrent release of the 6th generation Pomme magnetic model. Earth Planets Space 62:729-735

Maus S, Yin F, Lühr H, Manoj C, Rother M, Rauberg J, Michaelis I, Stolle C, Müller RD (2008) Resolution of direction of oceanic magnetic lineations by the sixth-generation lithospheric magnetic field model from CHAMP satellite magnetic measurements. Geochem Geophys Geosyst 9(7). doi:10.1029/2008GC001949

Olsen N, Tøffner-Clausen L, Sabaka TJ, Brauer P, Merayo JMG, Jørgensen JL, Léger J-M, Nielsen OV, Primdahl F, Risbo T (2003) Calibration of the Ørsted vector magnetometer. Earth Planets Space 55:11-18

Olsen N, Friis-Christensen E, Floberghagen R, Alken P, Beggan CD, Chulliat A, Doornbos E, da Encarnação JT, Hamilton B, Hulot G, van den IJssel J, Kuvshinov A, Lesur V, Lühr H, Macmillan S, Maus S, Noja M, Olsen PEH, Park J, Plank G, Püthe C, Rauberg J, Ritter P, Rother M, Sabaka TJ, Schachtschneider R, Sirol O, Stolle C, Thébault E, Thomson AWP, et al (2013) The Swarm Satellite Constellation Application and Research Facility (SCARF) and Swarm data products. Earth, Planets and Space 65(11):1189-1200. doi:10.5047/eps.2013.07.001

Peltier A, Chulliat A (2010) On the feasibility of promptly producing quasi-definitive magnetic observatory data. Earth Planets Space 62(2):5-8. doi:10.5047/eps.2010.02.002

Reigber C, Lühr H, Schwintzer P (2003) First CHAMP mission results for gravity, magnetic and atmospheric studies. Springer, Berlin

\section{Submit your manuscript to a SpringerOpen ${ }^{\odot}$ journal and benefit from:}

- Convenient online submission

Rigorous peer review

- Immediate publication on acceptance

- Open access: articles freely available online

- High visibility within the field

- Retaining the copyright to your article

Submit your next manuscript at $>$ springeropen.com 